\title{
The Importance of Emotional Intelligence in Nursing Care
}

\section{Diane E. White* PhD, RN, Sharon Grason PhD, RN}

School of Health Sciences, Georgia Gwinnett College, 1000 University Center Lane, Lawrenceville, Georgia 300043, United States of America.

\author{
Article Details \\ Article Type: Review Article \\ Received date: $07^{\text {th }}$ November, 2019 \\ Accepted date: $21^{\text {st }}$ November, 2019 \\ Published date: $23^{\text {rd }}$ November, 2019
}

"Corresponding Author: Diane E. White, School of Health Sciences, Georgia Gwinnett College, 1000 University Center Lane, Lawrenceville, Georgia 300043, United States of America. E-mail: Dwhite9@ggc.edu

Citation: White DE (2019) The Importance of Emotional Intelligence in Nursing Care. J Comp Nurs Res Care 4: 152. doi: https://doi.org/10.33790/jenrc1100152.

Copyright: (C2019, This is an open-access article distributed under the terms of the Creative Commons Attribution License 4.0, which permits unrestricted use, distribution, and reproduction in any medium, provided the original author and source are credited.

\begin{abstract}
This article reflects on how professional nurses learn caring behaviors in nursing school and then transpose those behaviors in the clinical setting. Although nursing is considered to be one of the most 'trusted' professions and a 'caring for others' profession, often there is a disconnect between this and actual experiences of patients and families in the clinical setting. Nurturing professional growth through emotional intelligence will be explored and application of emotional intelligence concepts will be applied to the profession of nursing. The authors contends that by fostering characteristics of emotional intelligence, nurses will not only be thought of as 'caring' but will also demonstrate behaviors that 'reconnect' the patient's perception to reality of the care experience.
\end{abstract}

Key words: Nursing, Care, Emotional Intelligence

\section{Introduction}

It is an assumption that professional nurses are caring and demonstrate such behaviors to patients and families; however, anecdotal patient and family stories of their experiences with professional nurses do not always support this premise. Maay, Ester, \&Arwam [1] found that caring behaviors by nurses influenced patient satisfaction levels. Additionally, nurses who utilized a patientcentered approach, fostered a sense of 'presence' to the patient that led to better patient outcomes [2]. Nurses who do not display this approach may be perceived as uncaring. The origin of these uncaring behaviors could be a result of experiences by the nurses in their nursing education.

Historically the educational environment has been decorated with behaviors that may appear as 'emotional hazing' to nursing students. Verbal and written communication is often laced with punitive words and negative connotations, such as 'fail' and 'not allowed.' Constant repetition of negative words leds to insecurity and no empowerment of the student nurse. These negative behaviors are learned and transcend into the practice environment where professional nurses 'eat their young' and has been labeled, 'bullying, incivility, and/or horizontal workplace violence' [3].

Often these behaviors go unreported due to fear of retaliation, and lack of administrative support [4]. These behaviors lead to physical and psychological oppression that cause many professional nurses to leave their workplace. These exits contribute to the shortage already noted in the workforce [4]. Instead of repeating these behaviors, leaders should intervene and expect accountability from professional nurses and coach them on how to 'care for' and 'care about' others $[5,6]$. By doing this, nurses will be able to better care for themselves, their colleagues and patients. The benefits to an organization by having patients and families feel they were cared for and cared about can be extraordinary.

The Joint Commission [7] and American Nurses Association [8] both require institutions to have policies and procedures in place that promote a culture of safety for health care team members and nurses. This culture is one where employees are treated with respect, is free of incivility and bullying behaviors, and workplace violence. In order to have this culture, nurses and other healthcare professionals need to be coached and mentored on developing this kind of culture.

Coaching caring behaviors can be done with intentional efforts toward nurturing professional growth based on emotional intelligence. Emotional intelligence (EI) in healthcare professionals has been studied and particularly with nursing $[9,10]$. Lack of emotional intelligence results in poor communication and uncivil behaviors such as bullying. Similarly, the Joint Commission [7] reported bullying behaviors linked to increased patient error, higher costs of care, higher turnover rates, lower patient satisfaction scores, and preventable adverse events. While the lack of emotional intelligence can cause negative behaviors, the presence of EI has positive effects. Toyama and Mauno [11] found nurses with traits of EI were more apt to have greater social support, work engagement, and creativity. Furthermore, nurses with EI traits were more productive at work and had greater job satisfaction.

\section{Methods}

An analysis of 'caring' demonstrates how caring is grounded in EI. Jenner [12] defined caring as '...intentional, creative use of oneself, based upon skill and expertise, to transmit meaning and emotion to another.' Nursing and caring are grounded in a relational understanding, unity, and connection between the professional nurse and patient.

There are five traits of EI: self-awareness, self-regulation, motivation, social awareness (empathy), and relationship management (social skills) [13]. Nurses with self-awareness know how they feel at all times and know how their emotions and actions can affect others.

Nurse leaders who have self-awareness have a clear picture of their strengths and weaknesses and behave with humility [14]. Being self-aware allows personal growth through the embracement of constructive feedback. Authentic self-awareness allows the nurse to 
identify personal strengths and weaknesses and to be comfortable with emotional discomfort. For example, a nurse with self-awareness may have different opinions with a colleague, but does not take the differences in opinion personally but rather acknowledges and embraces differences. This sounds easy to do; however, most people, not just nurses, would find this to be the most difficult trait of EI.

Self-awareness can be developed through objectively evaluating oneself [15]. Writing down one's strengths and weakness, helps oneself become more aware of themselves. Additionally, being willing to listen to colleagues providing honest and constructive feedback will promote personal and professional growth. Journaling this information along with one's achievements, goals, and plans will allow for self-reflection which fosters self-awareness. Mindfullness and mediation allows individuals to be at a place to better self-reflect and focus on self-awareness. Finally, personality tests exist that may help individuals better learn about traits that influence who they are and thus assist nurses in understanding why they feel and react the way they do personally and professionally.

Being self-aware allow nurses to be self-regulated, a second trait of emotional intelligence. Self-regulated nurses rarely verbally attack others, make rushed or emotional decisions, stereotype people, or compromise their values [16]. Self-regulation is all about staying in control. Although nurses find themselves in stressful situations, developing self-regulation will allow stressful situations to not become worse and minimize chaos. Self-regulation can be most difficult when interacting with colleagues who do not agree and patients and families who are in denial about their illness/wellness state. Nurses who self-regulate listen more than talk. They do not take other's comments personally, but rather puts the statements in context of the situation.

Self-regulation is not just about verbal restraint but also behavior surveillance. When interacting with colleagues, patients, and families it is important to be mindful of body language and the perceptions of others related to our body language. Often nurses are in a hurry due to multiple time sensitive tasks and do not realize how their lack of engagement with others effect the patient care experience. Slowing down, standing still, without hands crossed, making eye contact when dialoguing with others, will facilitate a positive perception of care by nurses. Being self-aware and self-regulating will help others feel cared for.

Self-regulation can be fostered by practicing mindfulness and meditation as noted above, along with identifying stressors in various areas of one's life. Knowing what triggers stress in one's life will help to regulate one's emotions and reactions when those stressors occur. Reflecting on instances when you did not self-regulate through journaling or talking with a mentor can help you to learn and move toward better self-regulation in the future. Asking questions such as, "Why am I or others feeling angry, frustrated, anxious, with this situation?" and "Are these emotions in play being directed at the right person or situation and to the right degree?" [15]. Reflecting on the answers to these questions can assist in the prevention of repeating the same mistake.

Motivation is another trait of emotional intelligence. People are not motivated by those who lash out, tear down or disempower others through lack of self-regulation. This is particularly true when speaking of nurses "eating their young" [16]. Often experienced nurses say things that cause the novice nurse to feel incapable of being a professional nurse and thus lessen his/her motivation to learn and grow in the profession. Experienced nurses who seek to motivate others will foster growth of others.

Not everyone is motivated by the same incentives. Some nurses will be intrinsically motivated while others will be extrinsically motivated. Taking the time to learn the uniqueness of one's motivation will assist you in fostering motivation. Some nurses will set personal goals of achievement (intrinsic), while others are motivated by hearing or receiving positive feedback (extrinsic); however, extrinsic motivation is not sustaining.

Fostering intrinsic motivation in others will sustain motivation. Assisting others in setting realistic goals and affirming the achievement of set goals will help others be motivated and find purpose in their work. Motivation can also be developed by using a '1:5 scorecard'. For every negative or non-motivating gesture done to others (self-awareness is needed), it takes 5 positive or motivating gestures to eliminate the negative. The goal is to have more positive gestures that foster motivation in others.

Another trait of EI is empathy. Empathy allows a nurse to put themselves in someone else's situation and be self-regulated. When nurses demonstrate empathy, nurses listen and do not react to emotional outbursts or deficiencies of other people. They are able to work in the context of understanding others in light of what others are experiencing [17].

Empathy can be developed by intentionally, cognitively putting oneself in another's shoes. For example, asking the other person, "How do you feel about that? What is on your mind?" To demonstrate true empathy for others one must also ask themselves, "What am I hearing the person say? Why does the person feel this way?" As the person begins to open up and deeper understanding is achieved, it is important to offer support.

Nurses often find themselves trying to 'rescue' or 'save' others and thus as nurses practice empathy it is important to be able to detach themselves from the person's experience while being empathetic. If unable to detach oneself, the nurse may find herself/himself with compassion fatigue. Compassion fatigue occurs when nurses become 'closed off ' or 'callous' to protect themselves from the pain other's experience by trying to be empathetic. Mindfulness meditation can help foster empathy. By being still, in the moment, allows one to be non-judgmental and accepting of what is, with clarity of heart [18] and assist the nurse in being able to detach and avoid compassion fatigue.

The last trait of EI is relationship management or social skills. Nurses who seek to have good relations with others exhibit EI and provide a canvas for others to feel cared for. Good relations must be intentional and not expected to organically happen. Nurses must seek to engage with others, not merely tolerate others. Engagement fosters communication and gives the opportunity to practice self-evaluation and self-regulation. When nurses engage with others, they are able to empathize as needed with others. Not engaging with others does not develop relationships and necessary social skills to be emotionally intelligent.

Often time you will hear people say, '...I just have to work with them, I don't have to like them or socialize with them.' While this may be true on some level; in order to give collaborative, patientcentered care, one has to have some type of relationship with his/her colleagues and patients. This is where EI comes into play and why it is important for nurses. Communication is the largest and most vital piece of social skills and relationship management.

Effective communicators not only place active listening as a priority but also exude warmth when talking with others. Warmth is felt by others when the communicator is friendly, approachable and is kind. In order to exhibit these attributes of warmth, the nurse needs to make sure that the verbal communication and non-verbal communication are parallel [19]. Assuming yourself as 'friendly' and saying the right words, but showing impatience or condescending behaviors while talking, dismisses the receiver from hearing the friendly words. Likewise, exhibiting warmth in non -verbal behaviors but choosing words that are demeaning, negative, judgmental and/or unkind will dismiss the warm behaviors evident in a conversation.

\section{Results}

Nurses with good relationship management and social skills not only 
demonstrate EI but also will find greater satisfaction in their work. Likewise, patient, families and colleagues who are exposed to nurses who possess EI will find their experiences much more satisfying. This allows both nurses and patients to flourish in their healthcare experiences. Nurses who intentionally develop their EI will allow their colleagues, patients and families to feel cared for and cared about.

\section{Conclusion}

As more nurses are exposed to EI in their nursing school education, they will be more inclined to exhibit the traits of EI in practice. Future recommendations are to have nurse educators begin incorporating $\mathrm{EI}$ in the pre and post licensure programs and for employers to offer educational offerings on the importance of EI in healthcare, specifically nursing. Future research could examine the patient's perceptions of EI when receiving nursing care and compare the results with the nurse's perception of their EI when providing care. Examining the similarities and differences may assist in fostering EI in facilitating positive patient experiences.

\section{References}

1. MaayJems KR, Ester R, Arwam H (2019) The relationship between nurse caring behavior and patient satisfaction level at inpatient wards of Bayangkara Hospital Jayapura. Indian Res Pub Health Res Dev 7: 1179-1183.

2. Rose P, Patsy P (2015) Patients' outcomes related to personcentered nursing care in radiation oncology: A case study. Eur J Oncol Nurs 19: 731-739.

3. Condon BB (2015) Incivility as bullying in nursing education. Nurs Sci Quarterly 28: 21-26.

4. Clark CM, Olender L, Kenski D, Cardoni C (2013) Exploring and addressing faculty to faculty incivility: A national perspective and literature review. J Nurs Edu 52: 211-218.

5. Clark CM, Springer PJ (2010) Academic nurse leaders' role in fostering a culture of civility in nursing education. J Nurs Edu 49: 319-325.

6. Kenner CA, Pressler JL (2014) Stress within the academic workplace. Nurs Edu 39: 105-106.

7. The Joint Commission (2008) Improving America's Hospitals: The Joint Commission's Annual Report on Quality and Safety.

8. American Nurses Association (2015) Code of Ethics for Nurses.

9. Lim SH, Han SS, Joo YS (2018) Effects of nurse's emotional intelligence on their organizational citizenship behavior, with mediating effects of leader trust and value congruence. J Nurs Sci 15: p363.

10. Basogul C, Ozgur G (2016) Role of emotional intelligence in conflict management strategies of nurses. Asian Nurs Res 10: 228-223.

11. Toyama H, Mauno S (2017) Associations of trait emotional intelligence with social support, work engagement, and creativity in Japanese eldercare nurses. Jpn Psychol Res 59: 1425.

12. Jenner (1997) The Art of Nursing: A concept analysis. Nurs forum 4: 5-11.

13. Bradberry T, Greaves J (2009) Emotional Intelligence 2.0. San Diego, CA: Talent Smart.

14. Seichter N (2018) Strengths-based stress and burnout prevention for nurse leaders. Nurs Leaders 4: 249-252.

15. Scott J (2019) What is self-awareness and how to develop it?

16. Meires J (2018) The Essentials: Using emotional Intelligence to curtail bullying in the workplace. Urol Nurs 38.

17. Stephany (2014) Cultivating Empathy: Inspiring Health Professionals to Communicate More Effectively.
18. Siegel R (2010) The Mindfulness Solution: Everyday Practices for Everyday Problems. New York: The Gulford Press.

19. Johnson WB, Ridley CR (2004) The traits of excellent mentors. The Elements of Mentoring 49-55. 